\title{
Controlling Molecular Assembly and Charge Transport of N-Type Organic Semiconductors with Sterically Demanding Substituents
}

\section{Craig Yu}

The University of Tokyo https://orcid.org/0000-0002-1423-5244

\section{Naoya Kojima}

The University of Tokyo

\section{Shohei Kumagai}

The University of Tokyo

\section{Tadanori Kurosawa}

The University of Tokyo

\section{Hiroyuki Ishii}

University of Tsukuba https://orcid.org/0000-0003-0644-1424

\section{Go Watanabe}

Kitasato University https://orcid.org/0000-0001-6713-1249

\section{Jun Takeya}

University of Tokyo https://orcid.org/0000-0001-7377-6043

\section{Toshihiro Okamoto ( $\nabla$ tokamoto@k.u-tokyo.ac.jp )}

The University of Tokyo

\section{Article}

Keywords: BQQDI, cyclohexyl-substituted

Posted Date: October 23rd, 2020

DOI: https://doi.org/10.21203/rs.3.rs-93683/v1

License: (c) (1) This work is licensed under a Creative Commons Attribution 4.0 International License. Read Full License

Version of Record: A version of this preprint was published at Communications Chemistry on November 11th, 2021. See the published version at https://doi.org/10.1038/s42004-021-00583-2. 


\section{Controlling molecular assembly and charge transport of n-type organic semiconductors with sterically demanding substituents}

Craig P. Yu ${ }^{1}$, Naoya Kojima ${ }^{2}$, Shohei Kumagai ${ }^{1}$, Tadanori Kurosawa ${ }^{1,2}$, Hiroyuki Ishii ${ }^{3}$, Go Watanabe $^{4}$, Jun Takeya ${ }^{1,2,5,6}$, and Toshihiro Okamoto ${ }^{1,2,5,7^{*}}$

${ }^{1}$ Material Innovation Research Center (MIRC) and Department of Advanced Materials Science, School of Frontier Sciences, The University of Tokyo, 5-1-5 Kashiwanoha, Kashiwa, Chiba 277-8561, Japan

${ }^{2}$ Department of Applied Chemistry, Faculty of Engineering, The University of Tokyo, 7-3-1 Hongo, Bunkyo-ku, Tokyo 113-0033, Japan

${ }^{3}$ Department of Applied Physics, Faculty of Pure and Applied Sciences, University of Tsukuba, 1-1-1 Tennodai, Tsukuba, Ibaraki 305-8573, Japan

${ }^{4}$ Department of Physics, School of Science, Kitasato University, 1-15-1 Kitasato, Minami-ku, Sagamihara, Kanagawa 252-0373, Japan.

${ }^{5}$ National Institute of Advanced Industrial Science and Technology (AIST)-University of Tokyo Advanced Operando-Measurement Technology Open Innovation Laboratory (OPERANDO-OIL), AIST, 5-1-5 Kashiwanoha, Kashiwa, Chiba 277-8561, Japan ${ }^{6}$ International Center for Materials Nanoarchitectonics (MANA), National Institute for Materials Science (NIMS), 1-1 Namiki, Tsukuba 205-0044, Japan ${ }^{7}$ PRESTO, JST, 4-1-8 Honcho, Kawaguchi, Saitama 332-0012, Japan *Corresponding Author: Toshihiro Okamoto, tokamoto@k.u-tokyo.ac.jp 


\begin{abstract}
Benzo $[d e]$ isoquinolino[1,8-gh]quinolinetetracarboxylic diimide (BQQDI) n-type organic semiconductors demonstrate unique multi-fold intermolecular hydrogen-bonding interactions that lead to excellent aggregated structures, charge transports, and electron mobility. However, the robust intermolecular anchoring of BQQDI presents challenges for further fine-tuning molecular assemblies and organic semiconductor properties. Herein, we report the design and synthesis of two BQQDI derivatives with sterically demanding phenyl- and cyclohexylsubstituted BQQDI (Ph-BQQDI and $\left.\mathrm{Cy}_{6}-\mathrm{BQQDI}\right)$, where the two organic semiconductors show distinct molecular assemblies and degrees of intermolecular orbital overlaps. In addition, the difference in their packing motifs led to strikingly different band structures that give rise to contrasting charge-transport capabilities. As a result, $\mathrm{Cy}_{6}-\mathrm{BQQDI}$ shows excellent transistor performances in both single-crystalline and polycrystalline thin-film organic field-effect transistors.
\end{abstract}

\title{
Introduction
}

Charge transport that gives rise to electrical properties of organic semiconductors (OSCs) is typically governed by intermolecular orbital overlaps and controlling such intermolecular interactions to achieve effective charge-transport properties lies in the center of molecular design for high-performance $\mathrm{OSCs}^{1,2}$. In the past decades, intense investigations of highperformance OSCs in terms of molecular design and device engineering fueled the rapid development of applicable organic-based electronic devices such as organic field-effect transistors (OFETs) $)^{3-5}$, which offer mechanical flexibility and low-cost processing compared to traditional inorganic-based devices. In particular, the hole-transporting p-type OSCs have shown promising OFET performances with charge-carrier mobilities $(\mu)$ over $10 \mathrm{~cm}^{2} \mathrm{~V}^{-1} \mathrm{~s}^{-1}$, and not only do these materials lead to applicable devices, they also provided crucial information on charge transport and guidance for future molecular designs ${ }^{6-13}$. On the other hand, the electron-transporting n-type OSCs, which are an essential component for constructing organic-based logic circuits ${ }^{14-16}$, are generally inferior to state-of-the-art p-type OSCs in terms of $\mu$. One of the challenges associated with the molecular design of n-type OSCs is that, owing to the energetics of charge injections and carrier transport, the lowest unoccupied molecular orbital (LUMO) level of n-type OSCs needs to be sufficiently low $(<-4.0 \mathrm{eV})$ to protect charge carriers from oxidation by ambient oxygen and moisture ${ }^{17,18}$. While the airstability issue of n-type OSCs can be addressed by incorporations of electron-deficient moieties ${ }^{19-21}$ and several studies have reported air-stable n-type OSCs with encouraging OFET 
performances $^{22-25}$, design strategies that focus on effective intermolecular orbital overlaps (quantified by transfer integral $t$ and effective mass $\left.m^{*}\right)^{26,27}$ and molecular assemblies for achieving favorable charge-transport properties and high electron mobility $\left(\mu_{\mathrm{e}}\right)$ are still required.
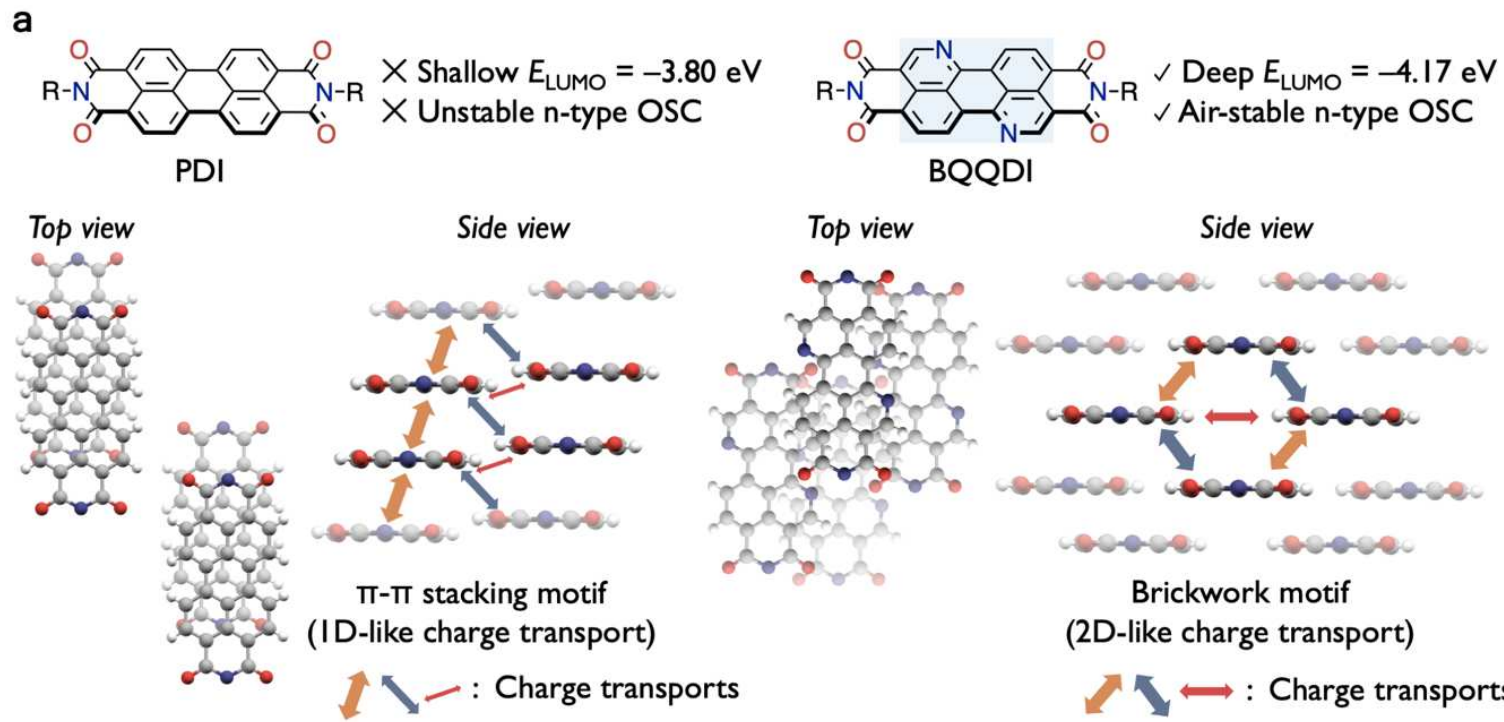

b

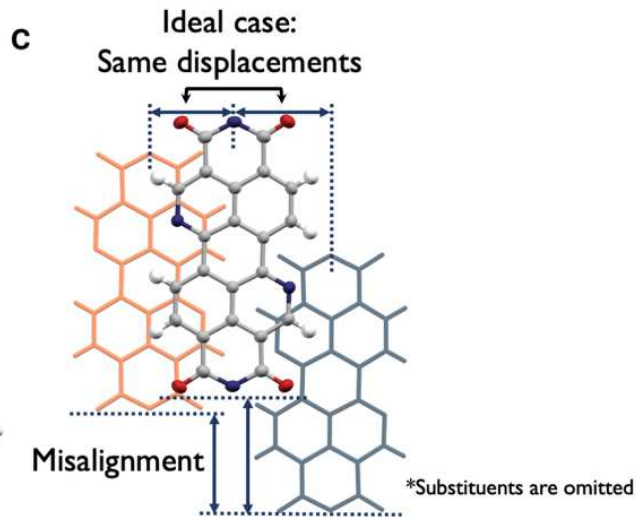

Fig. 1 Molecular features of $\mathbf{P h C}_{2}-\mathbf{B Q Q D I}$. a Structural, packing motif and charge transport comparisons between PDI and $\mathrm{BQQDI}$; b Intermolecular interactions of $\mathrm{PhC}_{2}-\mathrm{BQQDI}$; Molecular misalignment of $\mathrm{PhC}_{2}-\mathrm{BQQDI}$.

Recently, our group reported an air-stable and high-performance benzo[de]isoquinolino[1,8$g h$ ] quinolinetetracarboxylic diimide (BQQDI) $\pi$-electron $\operatorname{core}^{28}(\pi$-core) (Fig. 1a). The BQQDI is structurally analogous to the widely studied perylenetetracarboxylic diimide (PDI) system (Fig. 1a), though the electronegative nitrogen atoms in the BQQDI framework result in a DFT calculated deep-lying LUMO level of $-4.17 \mathrm{eV}$ (at the B3LYP/6-31G+(d) level ${ }^{29}$ for potential air-stable n-type charge transports, whereas the PDI $\pi$-core possesses a shallower LUMO level of $-3.80 \mathrm{eV}$. Upon functionalization of the BQQDI $\pi$-core with phenethyl $\left(\mathrm{PhC}_{2}-\mathrm{BQQDI}\right)$ groups, multi-fold hydrogen-bonding interactions are formed between adjacent molecules in 
the transverse direction (Fig. 1b), and strong $\pi-\pi$ interactions are also observed in the vertical direction. The resulting brickwork packing motifs show large $t$ values which indicate twodimensional (2D)-like charge-transport properties, whereas simple PDI derivatives generally exhibit one-dimensional (1D) $\pi-\pi$ stacking motif ${ }^{30,31}$ that leads to anisotropic charge-transport capabilities. The $\mathrm{PhC}_{2}-\mathrm{BQQDI}$ derivative, forms favorable phenyl-to-phenyl edge-to-face interactions between each molecular layer (Fig. 1b), in addition to the aforementioned intermolecular features, which significantly reinforce the intermolecular orbital overlaps as well as suppression of molecular motions. As a result, $\mathrm{PhC}_{2}-\mathrm{BQQDI}$ exhibits an impressive $\mu_{\mathrm{e}}$ of $3.0 \mathrm{~cm}^{2} \mathrm{~V}^{-1} \mathrm{~s}^{-1}$ in solution-processed OFETs, and excellent robustness against thermal- and bias-stress, which are necessary features for practical organic electronic applications.

Despite the encouraging results of $\mathrm{PhC}_{2}-\mathrm{BQQDI}$-based OSCs, the robust core-to-core and interlayer intermolecular interactions also pose challenges to further fine-tune molecular assemblies and charge-transport properties of BQQDI derivatives. By examining the packing structure of $\mathrm{PhC}_{2}-\mathrm{BQQDI}$, we notice that the hydrogen-bonding interactions cause some degree of misalignment in the $\pi-\pi$ stacking direction, which lead to an unbalanced chargetransport capability reflected by its $t$ and $m^{*}$ values. Herein, we report the investigation of two BQQDI derivatives with phenyl (Ph-BQQDI) and cyclohexyl ( $\left.\mathrm{Cy}_{6}-\mathrm{BQQDI}\right)$ substituents on their molecular assemblies and charge-transport capabilities. From a chemical perspective, we envisage that the installment of these sterically demanding substituents close to the BQQDI $\pi$ core compared to $\mathrm{PhC}_{2}-\mathrm{BQQDI}$ may sufficiently weaken the hydrogen-bonding interactions in the transverse direction and reduce the misalignment in intermolecular orbital overlaps. Owing to the different geometric and electronic properties, $\mathrm{Ph}-\mathrm{BQQDI}$ and $\mathrm{Cy}_{6}-\mathrm{BQQDI}$ exhibit distinct intra and interlayer molecular assemblies that lead to contrasting chargetransport capabilities as well as OSC performances. 


\section{Results and Discussion}

a

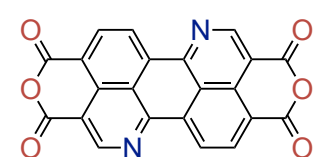

BQQ-TCDA

b

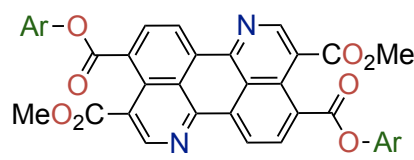

BQQ-TCP<smiles>Clc1cc(Cl)c(I=[Te])c(Cl)c1</smiles>

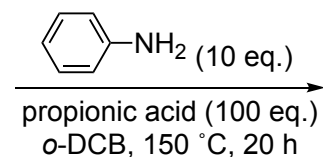

o-DCB, $150{ }^{\circ} \mathrm{C}, 20 \mathrm{~h}$

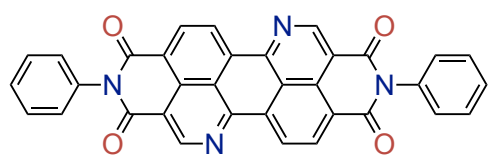

$\mathrm{Ph}-\mathrm{BQQDI}$

$91 \%$ yield

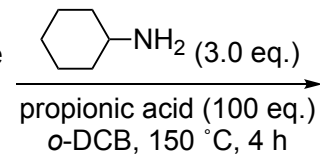

o-DCB, $150{ }^{\circ} \mathrm{C}, 4 \mathrm{~h}$

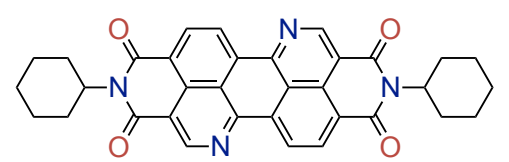

$\mathrm{Cy}_{6}-\mathrm{BQQDI}$

$94 \%$ yield

Fig. 2 Synthetic routes for BQQDI derivatives. a Synthesis of $\mathrm{Ph}-\mathrm{BQQDI}$ from BQQTCDA; b Synthesis of $\mathrm{Cy}_{6}$-BQQDI from BQQ-TCP.

The first target compound Ph-BQQDI was synthesized from the benzo[de]isoquinolino[1,8$g h$ ]quinolinetetracarboxylic dianhydride (BQQ-TCDA) starting material according to the previously reported procedure ${ }^{28}$ in $91 \%$ yield (Fig. 2a). However, formation of $\mathrm{Cy}_{6}-\mathrm{BQQDI}$ could only reach 70\% from BQQ-TCDA, along with 5\% mono-functionalized intermediate and $25 \%$ remaining starting material, likely due to lowering of the reactivity of amine by the sterically demanding cyclohexyl moiety. As reported before, the synthesis of 4-heptylsubstituted BQQDI (4-Hep-BQQDI) with bulky branched alkyl chains afforded only 10\% yield by using BQQ-TCDA as the starting material. To circumvent this issue, we discover that the precursor of BQQ-TCDA, 3,9-dimethyl-4,10-bis(2,4,6trichlorophenyl)benzo[de]isoquinolino[1,8-gh]quinoline-3,4,9,10-tetracarboxylate (BQQTCP) can also act as a viable starting material for the synthesis of BQQDI derivatives. The electrophilic trichlorophenyl formate groups ${ }^{32}$ of BQQ-TCP provides high reactivity towards imide condensation and tolerance to the somewhat bulky cyclohexyl amine. The $\mathrm{Cy}_{6}-\mathrm{BQQDI}$ target compound was successfully furnished from BQQ-TCP in 94\% yield (Fig. 2b), and 4Hep-BQQDI was also generated in $86 \%$ yield using the same procedure. 
a
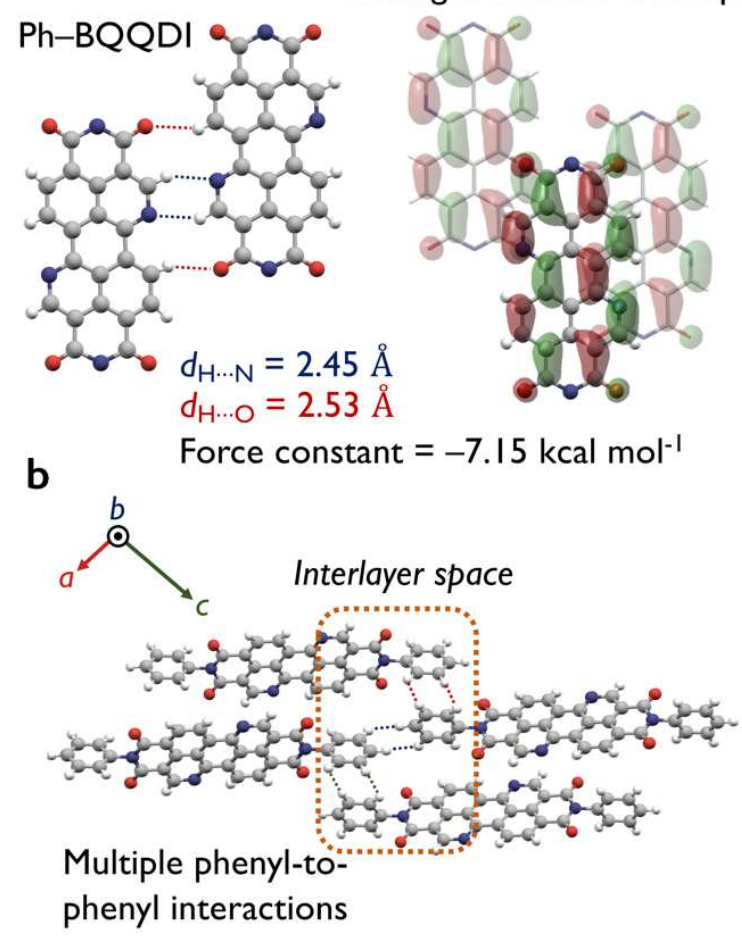

C

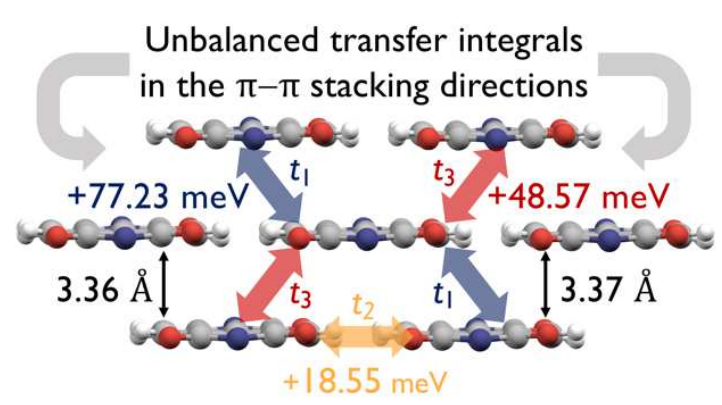

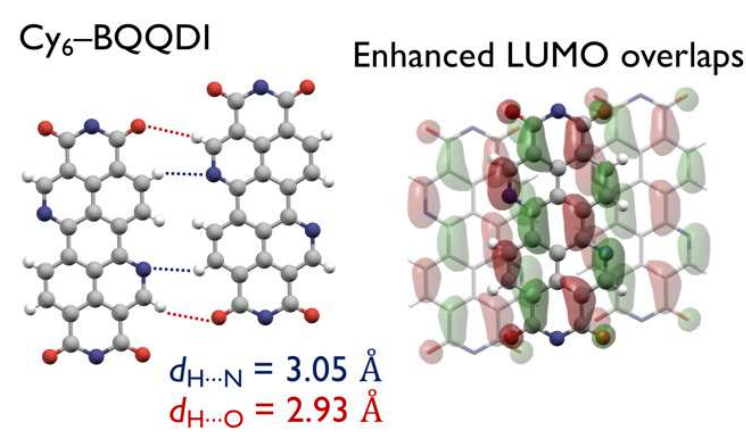

Force constant $=-2.20 \mathrm{kcal} \mathrm{mol}^{-1}$

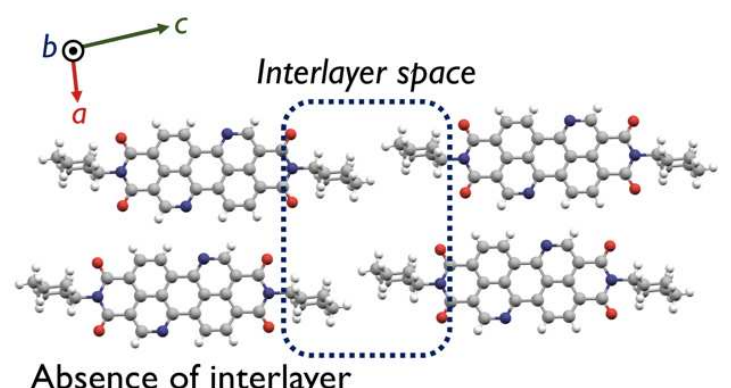
interactions

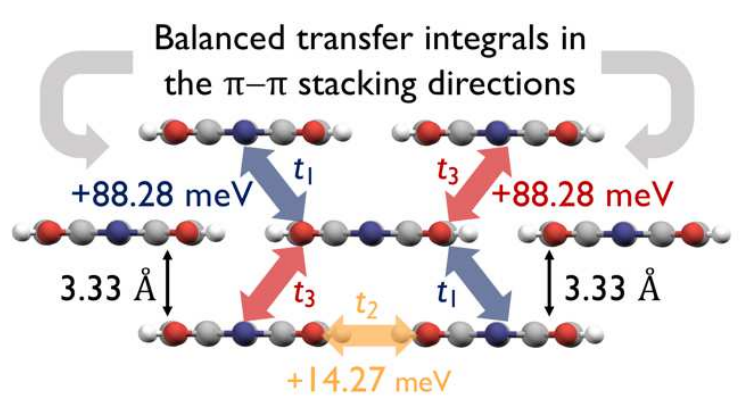

Fig. 3 Molecular assemblies and charge-transport capabilities of $\mathrm{Ph}-\mathrm{BQQDI}$ and $\mathrm{Cy}_{6}-$

BQQDI. a Dimer distances, interactions and LUMO overlaps; b Interlayer interactions; c Stacking distances and transfer integrals.

The single crystals of $\mathrm{Ph}-\mathrm{BQQDI}$ and $\mathrm{Cy}_{6}-\mathrm{BQQDI}$ were prepared using physical vapor transport (PVT) and solution-grown methods, respectively, and large plate-like crystals were obtained for both compounds (Fig. S5). Ph-BQQDI crystallizes in the monoclinic $P 2{ }_{1} / c$ space group with a 2D brickwork packing motif. Each planar BQQ $\pi$-core forms a four-fold hydrogen-bonding interactions with $\mathrm{O} \cdots \mathrm{H}$ and $\mathrm{N} \cdots \mathrm{H}$ short contacts on each side with its adjacent molecules in the transverse direction, along with slipped $\pi-\pi$ stacking interactions (Fig. 3a). The substituents of Ph-BQQDI form multiple phenyl-to-phenyl interactions in the interlayer space (Fig. 3b), which has been shown to be a favorable feature for suppressing molecular fluctuations ${ }^{28}$. Within the brickwork assembly of Ph-BQQDI, distances of the $\pi$ - 
stacks are found to be $3.36 \AA$ and $3.37 \AA$ (Fig. 3c), and the slight difference in distances is attributed to the misalignment between adjacent molecules in the transverse direction. The molecular assembly of Ph-BQQDI leads to a misalignment of LUMO of molecules in the $\pi-\pi$ stacking direction (Fig. 3a), where only a small degree of LUMO overlaps is observed between the top molecule and the molecule in the bottom layer. By calculating the $t$ values of $\mathrm{Ph}-$ BQQDI based on its crystal structure, it is evident that the misalignment in the assembly leads to different degrees of orbital overlaps with $t_{1}$ and $t_{3}$ equal to $+77.23 \mathrm{meV}$ and $+48.57 \mathrm{meV}$, respectively (Fig. 3c). Strong transverse interactions between $\pi$-cores are quantified by $t_{2}$ values of $+18.55 \mathrm{meV}$. By comparing the $t$ values of $\mathrm{Ph}-\mathrm{BQQDI}$ with the high-performance $\mathrm{PhC}_{2}-\mathrm{BQQDI}\left(t_{1}=+90.71 \mathrm{meV}, t_{2}=+18.89 \mathrm{meV}, t_{3}=+58.47 \mathrm{meV}\right)$, the $t$ values of Ph-BQQDI are much smaller than those of $\mathrm{PhC}_{2}-\mathrm{BQQDI}$, especially in the $\pi$ - $\pi$ stacking direction.

The $\mathrm{Cy}_{6}-\mathrm{BQQDI}$ derivative crystallizes in the monoclinic $C 2 / m$ space group with more symmetry than that of $\mathrm{Ph}-\mathrm{BQQDI}$. The steric bulk of cyclohexyl substituents likely prevents close contact between molecules in the transverse direction, which shows $\mathrm{O} \cdots \mathrm{H}$ and $\mathrm{N} \cdots \mathrm{H}$ interactions of $2.93 \AA$ and $3.05 \AA$, respectively, that are larger in distances than those between $\mathrm{Ph}-\mathrm{BQQDI}$ dimers. Force constant calculations of the transverse dimers at the M06-2X/6$31++\mathrm{G}(\mathrm{d}, \mathrm{p})$ level $^{33}$ further substantiate that $\mathrm{Cy}_{6}-\mathrm{BQQDI}$ shows a much weaker interaction energy of $-2.20 \mathrm{kcal} \mathrm{mol}^{-1}$ than that of Ph-BQQDI (-7.15 kcal mol${ }^{-1}$ ) (Fig. 3a). However, transverse dimers of $\mathrm{Cy}_{6}$-BQQDI show a much smaller displacement in the long molecular axis direction than $\mathrm{Ph}-\mathrm{BQQDI}$ dimers, and the reduced molecular misalignment of $\mathrm{Cy}_{6}-$ BQQDI leads to a much more enhanced LUMO overlaps in the $\pi-\pi$ stacking directions. In contrast to $\mathrm{Ph}-\mathrm{BQQDI}, \mathrm{Cy}_{6}-\mathrm{BQQDI}$ does not show any apparent interactions in the interlayer space (Fig. 3b), and we speculate that this may lead to larger molecular fluctuations of $\mathrm{Cy}_{6}-$ BQQDI than $\mathrm{Ph}-\mathrm{BQQDI}$. The 2D brickwork motif of $\mathrm{Cy}_{6}-\mathrm{BQQDI}$ shows a uniform $\pi-\pi$ stacking distance of $3.33 \AA$, which corresponds to the same degree of intermolecular orbital overlap with $t_{1}=t_{3}=+88.28 \mathrm{meV}$, which are larger than those of Ph-BQQDI. Even though the transverse dimer of $\mathrm{Cy}_{6}-\mathrm{BQQDI}$ demonstrates much weaker interaction energy than that of $\mathrm{Ph}-\mathrm{BQQDI}$ dimer, the transverse intermolecular orbital overlap of $\mathrm{Cy}_{6}-\mathrm{BQQDI}$ that is quantified by $t_{2}(+14.27 \mathrm{meV})$ is only slightly lower than that of Ph-BQQDI $(+18.55 \mathrm{meV})$ (Fig. 3c). The uniform charge-transport capability exhibited by $\mathrm{Cy}_{6}-\mathrm{BQQDI}$ may indicate promising OSC performances ${ }^{34}$. 


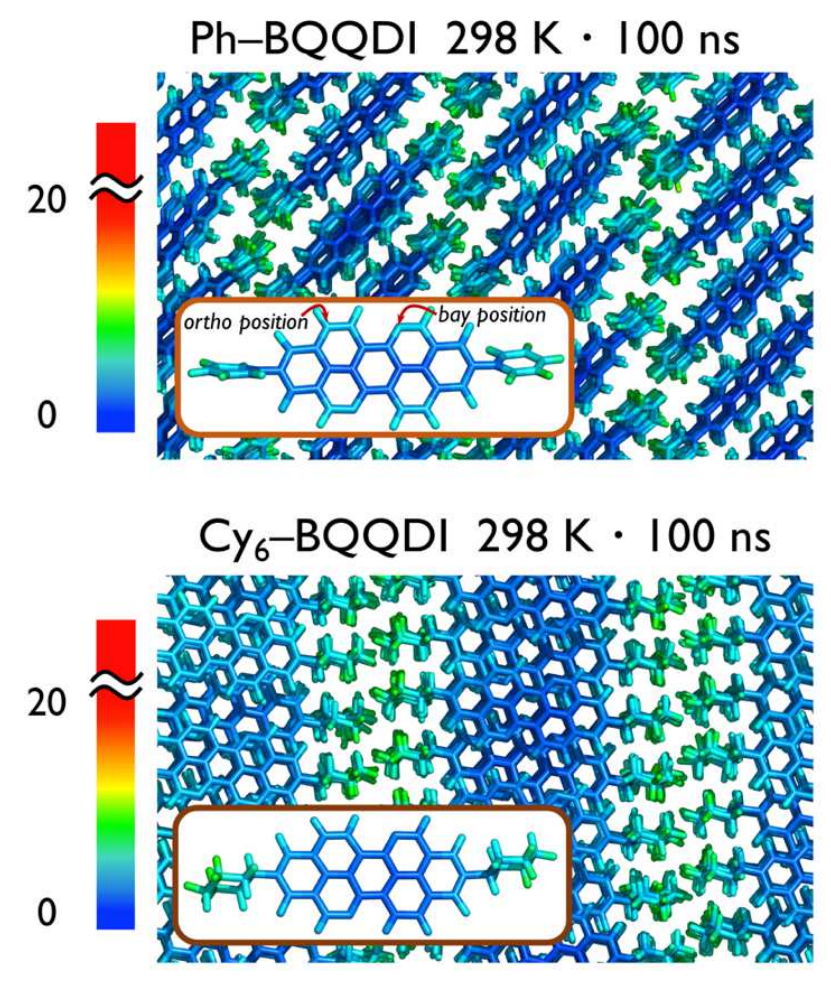

Fig. 4 Molecular simulations of Ph-BQQDI and Cy $\mathbf{y}_{6}-\mathrm{BQQDI}$. Color-coded B-factor distributions obtained from the trajectories during the last $10 \mathrm{~ns}$ of a $100 \mathrm{~ns}$ MD simulations in the NTP ensemble.

The different interlayer interactions of $\mathrm{Ph}-\mathrm{BQQDI}$ and $\mathrm{Cy}_{6}-\mathrm{BQQDI}$ prompted us to investigate their molecular fluctuations in the single-crystal state, and MD simulations with constant amount of substance $(\mathrm{N})$, temperature $(\mathrm{T})$, and pressure $(\mathrm{P})$ (isothermal-isobaric NTP ensemble) are performed based on their single-crystal structures measured at room temperature. $\mathrm{Ph}-\mathrm{BQQDI}$ with multiple phenyl-to-phenyl interactions in the interlayer space shows B-factor distributions similar to $\mathrm{PhC}_{2}-\mathrm{BQQDI}$, where small molecular fluctuations are observed on the substituents as well as the $\pi$-cores (Fig. 4). However, the atoms on the ortho and bay positions of Ph-BQQDI molecules show slightly larger B-factors than the rest of the $\pi$-core, which indicate that the rigid phenyl substituents and multiple interlayer interactions may not necessarily contribute to suppressed molecular fluctuations, rather the lack of flexibility in the substituents may contribute to some degree of structural perturbation. The B-factors of $\mathrm{Cy}_{6}-$ BQQDI are also similar to those of $\mathrm{PhC}_{2}-\mathrm{BQQDI}$ and $\mathrm{Ph}-\mathrm{BQQDI}$, where large B-factors are not observed on the ortho and bay position atoms (Fig. 4). Though, Cy 6 -BQQDI shows a unique case where some molecules have larger B-factors than others, and such a variant Bfactor distribution might be contributed to its lack of interlayer interactions as well as weak intermolecular interactions in the transverse direction. 

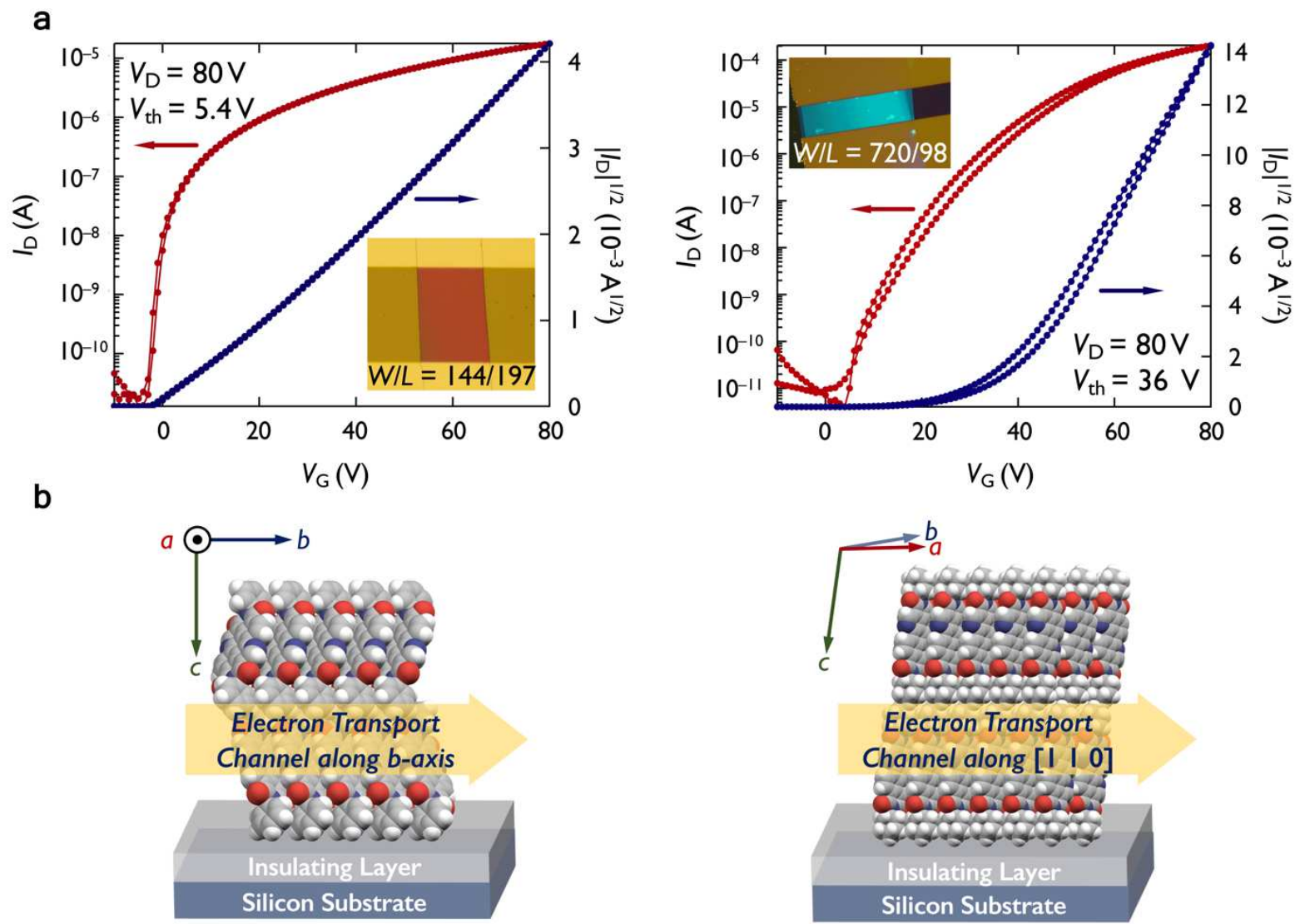

Fig. 5 OFET performances and thin film assemblies of Ph-BQQDI (left) and $\mathrm{Cy}_{6}-$ BQQDI (right). a Transfer characteristic of single-crystalline transistors; b Molecular assemblies in device states and corresponding channel directions.

To evaluate the $\mu_{\mathrm{e}}$ of $\mathrm{Ph}-$ and $\mathrm{Cy}_{6}-\mathrm{BQQDI}$, we fabricated bottom-gate/top-contact OFETs with gold electrodes using their single-crystalline thin films as the active OSC layer. Owing to the poor solubility of Ph-BQQDI, the PVT-grown single-crystalline thins films (193 nm thick) were directly laminated on a silicon substrate coated with a parylene insulating polymer, which has been used for laminated single-crystal OFETs ${ }^{35}$. The OSC single-crystalline thin films (7.79 nm thick) of the more soluble $\mathrm{Cy}_{6}-\mathrm{BQQDI}$ were prepared by the edge-casting method ${ }^{36}$ on the AL-X601-coated silicon substrate, which is an excellent insulating layer for solutionprocessed n-type single-crystalline thin-film OFETs ${ }^{28}$. The maximum $\mu_{\mathrm{e}}$ of $\mathrm{Ph}-\mathrm{BQQDI}$ was measured to be $1.0 \mathrm{~cm}^{2} \mathrm{~V}^{-1} \mathrm{~s}^{-1}$ (Fig. 5a). The highest $\mu_{\mathrm{e}}$ of $2.3 \mathrm{~cm}^{2} \mathrm{~V}^{-1} \mathrm{~s}^{-1}$ was achieved by the single-crystalline thin-film OFET using $\mathrm{Cy}_{6}-\mathrm{BQQDI}$ and an average $\mu_{\mathrm{e}}$ of $1.8 \pm 0.21 \mathrm{~cm}^{2} \mathrm{~V}^{-1}$ $\mathrm{s}^{-1}$ was measured over 12 devices (Fig. S7), and the devices showed excellent air-stability over one month (Fig. S8). Thin-film X-ray diffractions of the OSC active layers of $\mathrm{Ph}-$ and $\mathrm{Cy}_{6}-$ BQQDI reveal that their OFET channels directions correspond to the $b$-crystallographic axis and the $\left[\begin{array}{lll}1 & 1 & 0\end{array}\right]$ direction, respectively (Fig. S6). The molecular stacks of $\mathrm{Cy}_{6}-\mathrm{BQQDI}$ are 
roughly orthogonal to the OFET substrate with the $\pi$ - $\pi$ stackings that are parallel to the electron transport, whereas the molecular assembly of Ph-BQQDI creates more of an offset between the electron transport and the $\pi$ - $\pi$ stacking direction, which possibly leads to a less efficient electron transport.

Polycrystalline thin-film (40 nm thick) OFETs of $\mathrm{Ph}-$ and $\mathrm{Cy}_{6}-\mathrm{BQQDI}$ were also fabricated via vacuum deposition using decyltrimethoxysilane (DTS) as the self-assembled monolayer (SAM). The deposited thin film of Ph-BQQDI does not assume its single-crystal structure, as the $d$-spacing of $19.5 \AA$ at $2 \theta=4.52^{\circ}$ ( $d$-spacing $=15.5^{\circ}$ in single crystal) indicates a tilting angle of $24.5^{\circ}$ between the long axis of the molecules and the substrate (Fig. S11 and S13), which possibly originates from the interactions between the substrate and OSC molecules. The polycrystalline thin film of $\mathrm{Cy}_{6}-\mathrm{BQQDI}$ on the other hand, shows consistent molecular assembly as its single-crystal structure. Though, the diffraction peak at $2 \theta=17.04^{\circ}$ corresponds to the (1 1 -1) plane of the single-crystal structure of $\mathrm{Cy}_{6}-\mathrm{BQQDI}$, which indicates a thin-film orientational disordering ${ }^{37}$ with both edge-on and face-on-like stackings (Fig. S11 and S14). We evaluated the polycrystalline thin-film OFETs of $\mathrm{Ph}-$ and $\mathrm{Cy}_{6}-\mathrm{BQQDI}$, and the highest $\mu_{\mathrm{e}}$ of $0.16 \mathrm{~cm}^{2} \mathrm{~V}^{-1} \mathrm{~s}^{-1}$ was obtained for Ph-BQQDI (Fig. S15), which is one-order lower than its single-crystalline device, likely due to large grain boundary of the polycrystalline thin films (Fig. S10). Су ${ }_{6}-\mathrm{BQQDI}-$ based polycrystalline OFETs afforded the highest $\mu_{\mathrm{e}}$ of $0.50 \mathrm{~cm}^{2} \mathrm{~V}^{-1}$ $\mathrm{S}^{-1}$ on DTS (Fig. S16), and this promising result motivated us to explore other device conditions. When changed the SAM from DTS to hexamethyldisilazane (HMDS), which decreases the ratio of face-on/edge-on assemblies (Fig. S12), the highest $\mu_{\mathrm{e}}$ of $40 \mathrm{~nm}$-thick polycrystalline devices of $\mathrm{Cy}_{6}-\mathrm{BQQDI}$ was further improved to $0.66 \mathrm{~cm}^{2} \mathrm{~V}^{-1} \mathrm{~s}^{-1}$ (Fig. S14). We found that by reducing the $\mathrm{Cy}_{6}-\mathrm{BQQDI}$ OSC layer thickness from $40 \mathrm{~nm}$ to $20 \mathrm{~nm}$ on HMDS, an excellent highest $\mu_{\mathrm{e}}$ of $1.0 \mathrm{~cm}^{2} \mathrm{~V}^{-1} \mathrm{~s}^{-1}$ could be achieved, and the $\mu_{\mathrm{e}}$ appeared to be independent of the channel length (100-500 $\mu \mathrm{m})$ (Fig. S17-19). The device performances of polycrystalline $\mathrm{Cy}_{6}-$ BQQDI on DTS and HMDS in air are also consistent over more than one month (Fig. S20). The $\mu_{\mathrm{e}}$ of polycrystalline $\mathrm{Cy}_{6}-\mathrm{BQQDI}$ is one of the highest among current BQQDI derivatives, though, we speculate that its overall polycrystalline device performance might be hampered by the orientational disordering of its thin-film molecular assembly, and further optimization of the deposition conditions is currently undergoing. Both single- and polycrystalline OFETs based on $\mathrm{Cy}_{6}-\mathrm{BQQDI}$ show significantly higher $\mu_{\mathrm{e}}$ than those based on Ph-BQQDI, and the difference in their device performances are in agreement with their calculated $t$ values, but more in-depth analysis of their charge-transport capabilities is required. 


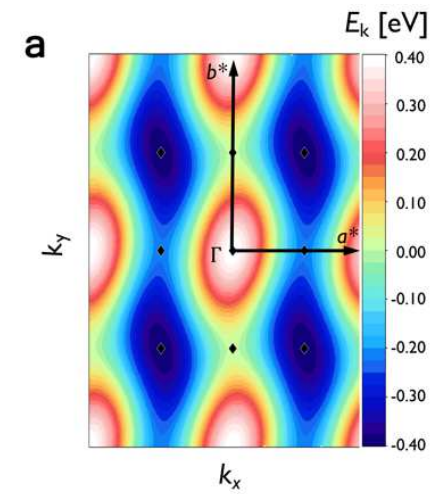

d

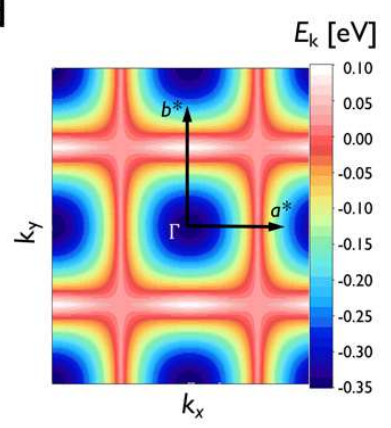

b

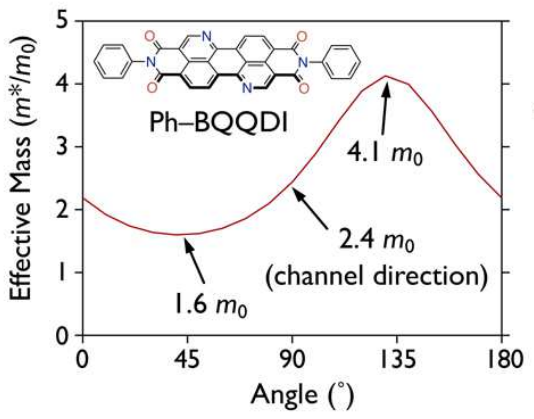

e

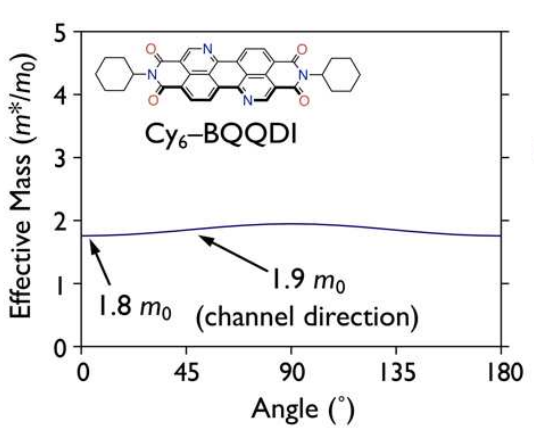

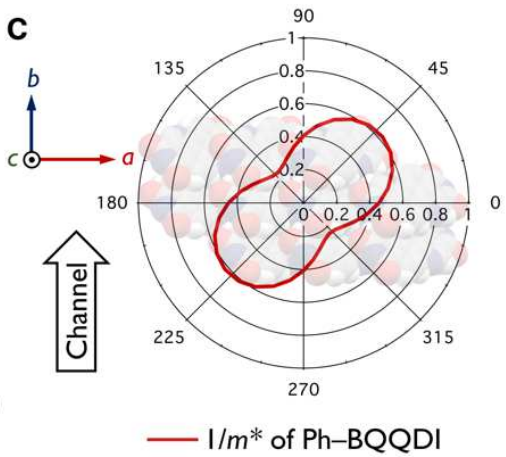

f

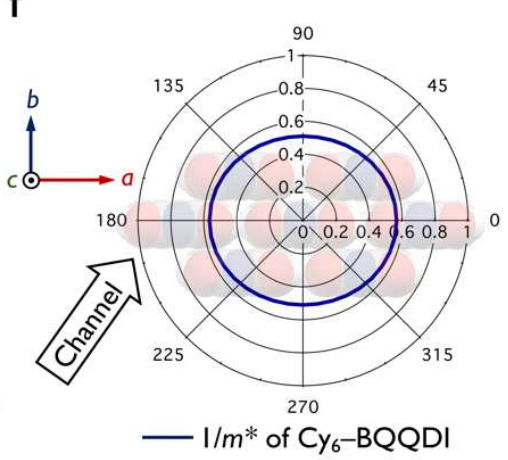

Fig. 6 Charge-transport capabilities calculated by the tight-binding approximation. a

and $\mathbf{d}$ 2D HOMO band; $\mathbf{b}$ and e Angle-resolved effective mass; $\mathbf{c}$ and $\mathbf{f}$ Angle-resolved inversed effective mass of $\mathrm{Ph}-\mathrm{BQQDI}$ and $\mathrm{Cy}_{6}-\mathrm{BQQDI}$, respectively.

We then further investigated the directionality of the charge-transport capabilities of $\mathrm{Ph}-$ and $\mathrm{Cy}_{6}-\mathrm{BQQDI}$ using the tight-binding approximation ${ }^{38}$ to rationalize the difference in their OFET performances. Ph-BQQDI exhibits elliptical shaped 2D LUMO bands, and from the bottom edge of the band dispersion (Fig. S21), we calculated the $m^{*}$ values with respect to the crystallographic axes. The smallest $m^{*}$ value of $\mathrm{Ph}-\mathrm{BQQDI}$ is $1.6 m_{0}$, which is found at $45^{\circ}$ from the $a$-axis ([ $\left.\begin{array}{lll}1 & 1 & 0\end{array}\right]$ direction). However, the OFET channel along the $b$-axis direction of $\mathrm{Ph}-\mathrm{BQQDI}$ corresponds to a larger $m^{*}$ of $2.4 m_{0}$. On the other hand, Cy 6 -BQQDI shows a more circular 2D LUMO band and the resulting $m^{*}$ values are seemingly uniform across the crystallographic directions. The $m^{*}$ of $\mathrm{Cy}_{6}-\mathrm{BQQDI}$ along the OFET channel direction is calculated to be $1.9 m_{0}$, which is only slightly larger than its smallest $m^{*}$ of $1.8 m_{0}$ in the $a$-axis direction. The directionality of the charge transport of the current BQQDI derivatives can be better visualized from the angle-resolved inversed $m^{*}$ plots, where $\mathrm{Ph}-\mathrm{BQQDI}$ shows a peanut shaped curve, with its best charge transport at $45^{\circ}$ relative to the $a$-axis ([ 1110$]$ direction), and poorer charge-transport capability is found in the $b$-axis (channel) direction. The inversed $m^{*}$ plot of $\mathrm{Cy}_{6}-\mathrm{BQQDI}$ shows a more uniform charge-transport behavior, where a favorable charge-transport capability can be found along the channel direction of its OFETs. The high 
polycrystalline device performance of $\mathrm{Cy}_{6}-\mathrm{BQQDI}$ despite having the orientationally disordered thin-film assembly could be attributed to its isotropic-like charge-transport capability. The current results suggest the importance of molecular design not only in the bulk crystal state, but also in the thin-film state along the channel direction for achieving high device performances.

In summary, the current work reports interesting distinct effects of two sterically demanding substituents on the molecular assemblies in both bulk crystal and thin-film states. Their crystal packing structures lead to different charge-transport capabilities, where the $\mathrm{Cy}_{6}-\mathrm{BQQDI}$ derivative exhibits uniform transfer integrals and effective mass compared to the Ph-BQQDI counterpart. Despite having no apparent interlayer interactions, the $\pi$-cores of $\mathrm{Cy}_{6}-\mathrm{BQQDI}$ do not show significantly larger molecular fluctuations than $\mathrm{Ph}-\mathrm{BQQDI}$. In fact, the rigid phenyl groups with multiple interlayer interactions might have led to noticeable fluctuations of the atoms in the bay positions. Though from the tight-binding approximations, the smallest effective mass of $\mathrm{Ph}-\mathrm{BQQDI}$ is smaller than that of $\mathrm{Cy}_{6}-\mathrm{BQQDI}$, the effective mass of $\mathrm{Cy}_{6}-$ BQQDI along the OFET channel direction is smaller than that of Ph-BQQDI. Thus, OFETs of $\mathrm{Cy}_{6}-\mathrm{BQQDI}$ exhibits an excellent $\mu_{\mathrm{e}}$ of $2.3 \mathrm{~cm}^{2} \mathrm{~V}^{-1} \mathrm{~s}^{-1}$ in single-crystalline thin film, and up to $1.0 \mathrm{~cm}^{2} \mathrm{~V}^{-1} \mathrm{~s}^{-1}$ in polycrystalline devices. The results herein demonstrate an effective molecular design for molecular assembly, charge transport, and suppressing molecular fluctuations in the bulk single-crystal state, as well as controlling the molecular assembly in the thin-film device state for achieving the optimum charge-transport capabilities. Future work based on the current encouraging results of $\mathrm{Cy}_{6}-\mathrm{BQQDI}$ may involve incorporations of alkyl substituents on the cyclohexyl group to improve its solubility for large-area device fabrications.

\section{Methods}

Materials and general characterizations. All amine reagents used in this study were purchased from Tokyo Chemical Industry Co., Ltd and propionic acid was purchased from FUJIFILM Wako Pure Chemical Industries, Ltd without further purifications. $o$ dichlorobenzene (o-DCB) was purchased from KANTO chemical Co., Ltd. and purified by a solvent purification system. Starting materials 3,4,9,10-benzo[de]isoquinolino[1,8gh]quinolinetetracarboxylic dianhydride (BQQ-TCDA) and 3,9-dimethyl-4,10-bis(2,4,6trichlorophenyl)benzo[de]isoquinolino[1,8-gh]quinoline-3,4,9,10-tetracarboxylate (BQQTCP) were synthesized and purified in our laboratory prior to this study. All reactions were carried out under an atmosphere of argon. ${ }^{1} \mathrm{H}$ NMR spectra were recorded on JEOL ECS400 spectrometer $(400 \mathrm{MHz})$. Chemical shifts were reported in parts per million (ppm, $\delta$ scale) 
from residual protons in the deuterated solvent for ${ }^{1} \mathrm{H}$ NMR (5.93 ppm for 1,1,2,2tetrachloroethane- $d_{2}\left(\right.$ TCE- $\left.d_{2}\right), 1,1,1,3,3,3$-hexafluoro-2-propanol- $d_{2}\left(\right.$ HFIP- $\left.d_{2}\right)$ and 7.26 ppm for chloroform- $\left.d\left(\mathrm{CDCl}_{3}\right)\right)$. The data were presented in the following format: chemical shift, multiplicity $(\mathrm{s}=$ singlet, $\mathrm{d}=$ doublet, $\mathrm{t}=$ triplet, quint $=$ quintet, $\mathrm{m}=$ multiplet $)$, coupling constant in Hertz (Hz), signal area integration in natural numbers. Time-of-flight high-resolution mass (TOF-MS) spectrometry measurements were measured on a BRUKER compact-TKP2 mass spectrometer with the atmospheric pressure chemical ionization (APCI) method. Elemental analysis measurements were carried out on a JScience Lab JM10 CHN analyzer at the Comprehensive Analysis Center, the Institute of Scientific and Industrial Research, Osaka University.

Synthesis. Detailed synthetic procedures, compound characterizations, and ${ }^{1} \mathrm{H}$ NMR of BQQDI derivatives are reported in the Supplementary Information.

Theoretical calculations. Estimations of transfer integral and effective mass were conducted using the GAMESS package ${ }^{39}$. The Kohn-Sham eigenstates of all compounds in this work were calculated at the PBEPBE/6-31G(d) level of theory. Transfer integrals between LUMOs of neighboring molecules in the crystal structures were estimated by the dimer method ${ }^{1}$. To further understand the charge-transport capabilities in the single-crystal state, their LUMO band structures $E(k)$ were calculated by the tight-binding approximation using transfer integrals. Intermolecular interaction energy between two adjacent molecules were obtained at the M06-2X/6-31++G(d,p) level of DFT with counterpoise correction for the basis set superposition error ${ }^{33}$. The calculations were performed using the Gaussian 09 program package $^{40}$.

X-ray crystallography. $\mathrm{Ph}-\mathrm{BQQDI}$ single crystals were obtained by means of PVT, and $\mathrm{Cy}_{6}-\mathrm{BQQDI}$ crystals were grown in the mixture of nitrobenzene and 1-chloronaphthalene via the slow-cooling method. Single-crystal X-ray diffraction data were collected on a Rigaku RAXIS RAPID II imaging plate diffractometer with $\mathrm{CuK} \alpha$ radiation $(\lambda=1.54187 \AA)$ at room temperature. The structures were solved by direct methods [SHELXT (2015)] and refined by full-matrix least-squares procedures on F2 for all reflections [SHELXL (Ver. 2014/7)]. While positions of all hydrogen atoms were calculated geometrically, and refined by applying riding model, all other atoms were refined anisotropically. Crystallographic data have been deposited in the Cambridge Crystallographic Data Centre as a supplementary publication. These data can be obtained free of charge at www.ccdc.cam.ac.uk/data_request/cif. 


\section{References}

1. Coropceanu, V. et al. Charge transport in organic semiconductors. Chem. Rev. 107, 926-952 (2007).

2. Fratini, S., Nikolka, M., Salleo, A., Schweicher, G. \& Sirringhaus, H. Charge transport in high-mobility conjugated polymers and molecular semiconductors. Nat. Mater. 19, 491-502 (2020).

3. Klauk, H. Organic thin-film transistors. Chem. Soc. Rev. 39, 2643-2666 (2010).

4. Wang, C., Dong, H., Hu, W., Liu, Y. \& Zhu, D. Semiconducting $\pi$-conjugated systems in field-effect transistors: A material odyssey of organic electronics. Chem. Rev. 112, 2208-2267 (2012).

5. Mei, J., Diao, Y., Appleton, A. L., Fang, L. \& Bao, Z. Integrated materials design of organic semiconductors for field-effect transistors. J. Am. Chem. Soc. 135, 6724-6746 (2013).

6. Ebata, H. et al. Highly soluble [1]benzothieno[3,2-b]benzothiophene (BTBT) derivatives for high-performance, solution-processed organic field-effect transistors. $J$. Am. Chem. Soc. 129, 15732-15733 (2007).

7. Yamamoto, T. \& Takimiya, K. Facile synthesis of highly $\pi$-extended heteroarenes, dinaphtho[2,3-b: 2',3'-f]chalcogenopheno[3,2-b]chalcogenophenes, and their application to field-effect transistors. J. Am. Chem. Soc. 129, 2224-2225 (2007).

8. Anthony, J. E. The larger acenes: Versatile organic semiconductors. Angew. Chem. Int. Ed. 47, 452-483 (2008).

9. Okamoto, T. et al. V-shaped organic semiconductors with solution processability, high mobility, and high thermal durability. Adv. Mater. 25, 6392-6397 (2013).

10. Mitsui, C. et al. High-performance solution-processable N-shaped organic semiconducting materials with stabilized crystal phase. Adv. Mater. 26, 4546-4551 (2014).

11. Yamamoto, A. et al. Zigzag-Elongated Fused $\pi$-Electronic Core: A Molecular Design Strategy to Maximize Charge-Carrier Mobility. Adv. Sci. 5, 1700317 (2018).

12. Okamoto, T. et al. Bent-Shaped p-Type Small-Molecule Organic Semiconductors: A Molecular Design Strategy for Next-Generation Practical Applications. J. Am. Chem. 
Soc. 142, 9083-9096 (2020).

13. Okamoto, T. et al. Alkyl-Substituted Selenium-Bridged V-Shaped Organic Semiconductors Exhibiting High Hole Mobility and Unusual Aggregation Behavior. $J$. Am. Chem. Soc. 142, 14974-14984 (2020).

14. Crone, B. et al. Large-scale complementary integrated circuits based on organic transistors. Nature 403, 521-523 (2000).

15. Klauk, H., Zschieschang, U., Pflaum, J. \& Halik, M. Ultralow-power organic complementary circuits. Nature 445, 745-748 (2007).

16. Smits, E. C. P. et al. Bottom-up organic integrated circuits. Nature 455, 956-959 (2008)

17. Usta, H., Facchetti, A. \& Marks, T. J. N-channel semiconductor materials design for organic complementary circuits. Acc. Chem. Res. 44, 501-510 (2011).

18. Zhou, K., Dong, H., Zhang, H. li \& Hu, W. High performance n-type and ambipolar small organic semiconductors for organic thin film transistors. Phys. Chem. Chem. Phys. 16, 22448-22457 (2014).

19. Jones, B. A., Facchetti, A., Wasielewski, M. R. \& Marks, T. J. Tuning orbital energetics in arylene diimide semiconductors. Materials design for ambient stability of n-type charge transport. J. Am. Chem. Soc. 129, 15259-15278 (2007).

20. Fukutomi, Y., Nakano, M., Hu, J. Y., Osaka, I. \& Takimiya, K.

Naphthodithiophenediimide (NDTI): Synthesis, structure, and applications. J. Am. Chem. Soc. 135, 11445-11448 (2013).

21. Yu, C. P. et al. Air-Stable Benzo[c]thiophene Diimide n-Type $\pi$-Electron Core. Org. Lett. 21, 4448-4453 (2019).

22. Molinari, A. S., Alves, H., Chen, Z., Facchetti, A. \& Morpurgo, A. F. High electron mobility in vacuum and ambient for PDIF-CN2 single-crystal transistors. J. Am. Chem. Soc. 131, 2462-2463 (2009).

23. Liang, Z., Tang, Q., Xu, J. \& Miao, Q. Soluble and Stable N-Heteropentacenes with High Field-Effect Mobility. Adv. Mater. 23, 1535-1539 (2011).

24. Xu, X. et al. Electron Mobility Exceeding $10 \mathrm{~cm} 2 \mathrm{~V}-1 \mathrm{~s}-1$ and Band-Like Charge Transport in Solution-Processed n-Channel Organic Thin-Film Transistors. Adv. 
Mater. 28, 5276-5283 (2016).

25. Vladimirov, I. et al. High-Mobility, Ultrathin Organic Semiconducting Films Realized by Surface-Mediated Crystallization. Nano Lett. 18, 9-14 (2018).

26. Brédas, J. L., Calbert, J. P., Da Silva Filho, D. A. \& Cornil, J. Organic semiconductors: A theoretical characterization of the basic parameters governing charge transport. Proc. Natl. Acad. Sci. U.S.A. 99, 5804-5809 (2002).

27. Valeev, E. F., Coropceanu, V., Da Silva Filho, D. A., Salman, S. \& Brédas, J. L. Effect of electronic polarization on charge-transport parameters in molecular organic semiconductors. J. Am. Chem. Soc. 128, 9882-9886 (2006).

28. Okamoto, T. et al. Robust, high-performance n-type organic semiconductors. Sci. Adv. 6, eaaz0632 (2020).

29. Becke, A. D. Density-functional thermochemistry. III. The role of exact exchange. $J$. Chem. Phys. 98, 5648-5652 (1993).

30. Mizuguchi, J. N,N'-bis(2-phenethyl)perylene-3,4:9,10-bis-(dicarboximide). Acta Crystallogr. Sect. C Cryst. Struct. Commun. 54, 1479-1481 (1998).

31. Krauss, T. N. et al. Three-dimensional molecular packing of thin organic films of PTCDI-C8 determined by surface X-ray diffraction. Langmuir 24, 12742-12744 (2008).

32. Ueda, T., Konishi, H. \& Manabe, K. Trichlorophenyl formate: Highly reactive and easily accessible crystalline CO surrogate for palladium-catalyzed carbonylation of aryl/alkenyl halides and triflates. Org. Lett. 14, 5370-5373 (2012).

33. Boys, S. F. \& Bernardi, F. The calculation of small molecular interactions by the differences of separate total energies. Some procedures with reduced errors. Mol. Phys. 19, 553-566 (1970).

34. Fratini, S., Ciuchi, S., Mayou, D., De Laissardière, G. T. \& Troisi, A. A map of highmobility molecular semiconductors. Nat. Mater. 16, 998-1002 (2017).

35. Pfattner, R. et al. High-performance single crystal organic field-effect transistors based on two dithiophene-tetrathiafulvalene (DT-TTF) polymorphs. Adv. Mater. 22, 41984203 (2010).

36. Uemura, T., Hirose, Y., Uno, M., Takimiya, K. \& Takeya, J. Very high mobility in 
solution-processed organic thin-film transistors of highly ordered [1]benzothieno[32b]benzothiophene derivatives. Appl. Phys. Express 2, 111501 (2009).

37. Gann, E., Gao, X., Di, C. A. \& McNeill, C. R. Phase transitions and anisotropic thermal expansion in high mobility core-expanded naphthalene diimide thin film transistors. Adv. Funct. Mater. 24, 7211-7220 (2014).

38. Kuroda, Y., Ishii, H., Yoshino, S. \& Kobayashi, N. Second highest occupied molecular orbital effects on the valence band structure of organic semiconductors. Jpn. J. Appl. Phys. 58, SIIB27 (2019).

39. Schmidt, M. W. et al. General atomic and molecular electronic structure system. $J$. Comput. Chem. 14, 1347-1363 (1993).

40. Frisch, M. J. et al. Gaussian 09, revision A. 02. Gaussian, Inc., Wallingford, CT, 2009 (1988).

\section{Acknowledgements}

The authors thank AGC Inc. for supplying AL-X601. The computation reported in this paper was performed at the Research Center for Computational Science, Okazaki, Japan. This work was supported by the JST-PRESTO program "Scientific Innovation for Energy Harvesting Technology" (number JPMJPR17R2) and by KAKENHI. T.O., H.I., G.W., and C.P.Y. thank JSPS for Grants-in-Aid for Scientific Research, B (numbers 17H03104, 18H01856, 19H02537, and 20J12608).

\section{Author contributions}

T.O. conceived and designed the work, while C.P.Y., N.K., S.K., T.K. synthesized the compounds. C.P.Y., S.K., and N.K. performed the physicochemical properties measurements, single-crystal and thin-film X-ray analyses, and OFET evaluations. H.I. and C.P.Y. calculated the transfer integrals and effective masses. G.W. performed the molecular dynamics simulations. C.P.Y. performed the DFT studies. C.P.Y. and T.O. wrote the manuscript. J.T. and T.O. supervised the work. All authors discussed the results and reviewed the manuscript prior to submission.

\section{Competing interests}

The authors declare no competing interests. 
Figures

a

$\times$ Shallow $E_{\text {LUMO }}=-3.80 \mathrm{eV}$
$\times$ Unstable n-type OSC

PDI $\checkmark$ Deep $E_{\text {LUMO }}=-4.17 \mathrm{eV}$
Air-stable n-type OSC

BQQDI

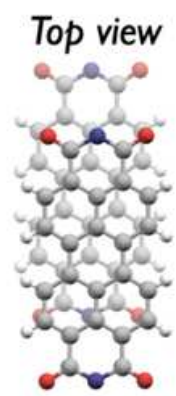

b

$$
\mathrm{PhC}_{2} \text {-BQQDI }
$$

Hydrogen bonding

(ID-like charge transport)

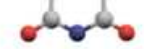

Side view

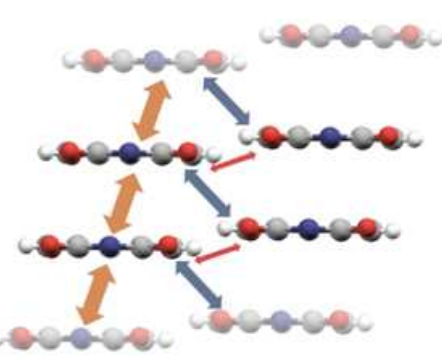

$\pi-\pi$ stacking motif

1 Charge transports
Top view

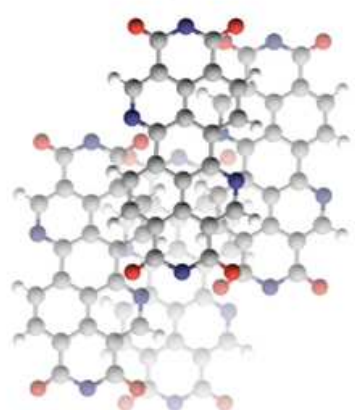

\section{Hydrogen bonding}

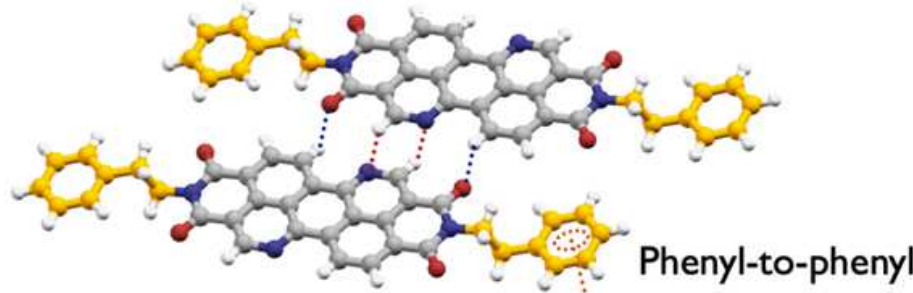

$\checkmark$ Lateral $\mathrm{H}$-bonding

$\checkmark$ Interlayer anchoring

$\checkmark 2 \mathrm{D}$ charge transport

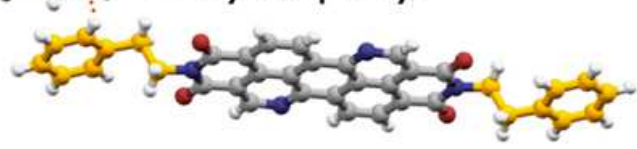

c

Ideal case:

Same displacements

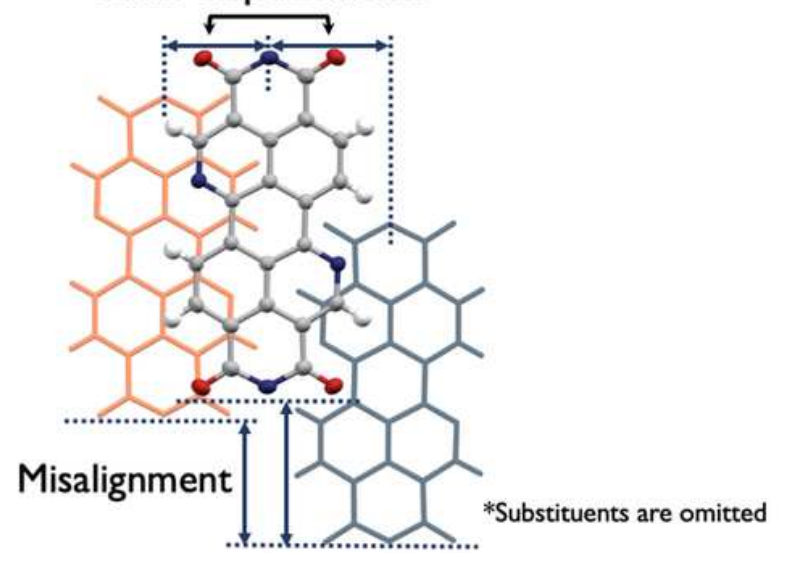

Figure 1

Molecular features of PhC2-BQQDI. a Structural, packing motif and charge transport comparisons between PDI and BQQDI; $b$ Intermolecular interactions of PhC2-BQQDI; c Molecular misalignment of PhC2-BQQDI. 
a<smiles>O=C1OC(=O)c2cnc3c4ccc5c6c(ccc(c7ccc(c2c73)C1=O)c64)C(=O)OC5=O</smiles>

BQQ-TCDA

b<smiles></smiles>

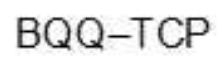<smiles>Clc1cc(Cl)c(I)c(Cl)c1</smiles>

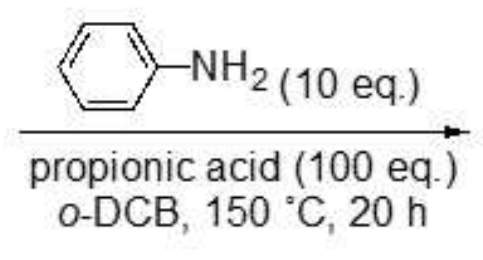<smiles>O=C1c2ccc3c4ccc5c6c(ccc(c7ccc(c2c37)C(=O)N1c1ccccc1)c64)C(=O)N(c1ccccc1)C5=O</smiles>

$\mathrm{Ph}-\mathrm{BQQDI}$ $91 \%$ yield

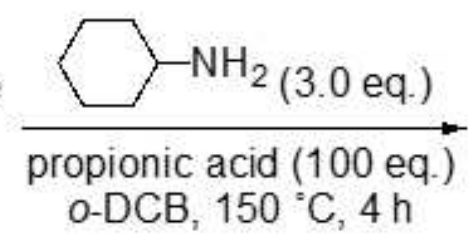

o-DCB, $150^{\circ} \mathrm{C}, 4 \mathrm{~h}$

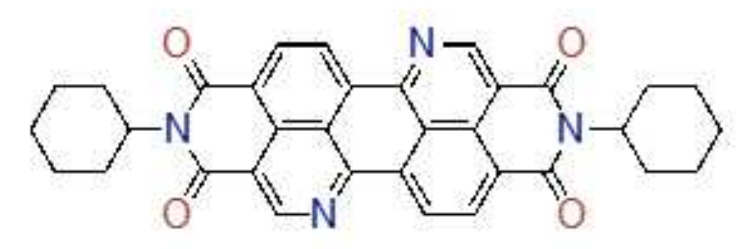

$\mathrm{Cy}_{6}-\mathrm{BQQDI}$

$94 \%$ yield

\section{Figure 2}

Synthetic routes for BQQDI derivatives. a Synthesis of Ph-BQQDI from BQQ-TCDA; b Synthesis of Cy6BQQDI from BQQ-TCP. 
a

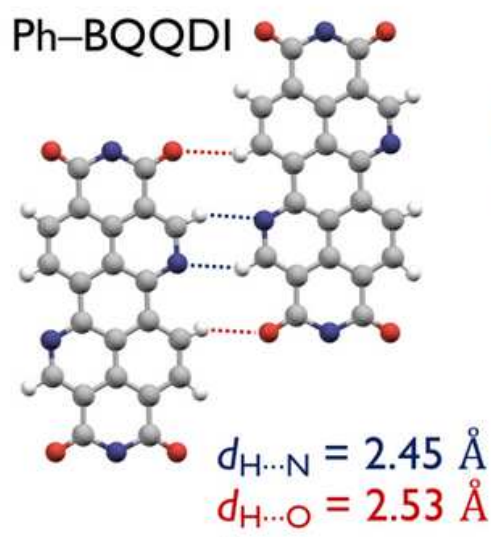

b

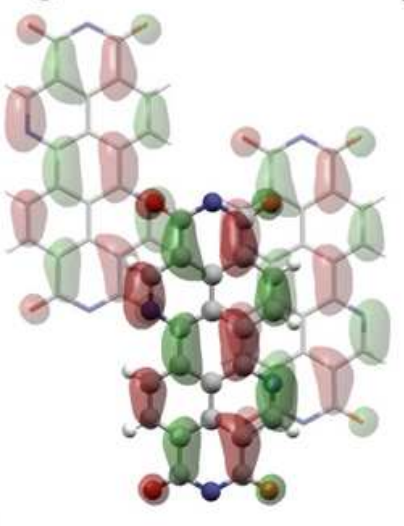

\section{$\mathrm{Cy}_{6}-\mathrm{BQQDI}$}

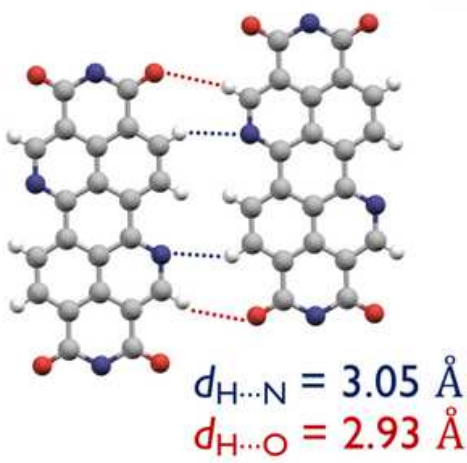

Force constant $=-2.20 \mathrm{kcal} \mathrm{mol}^{-1}$

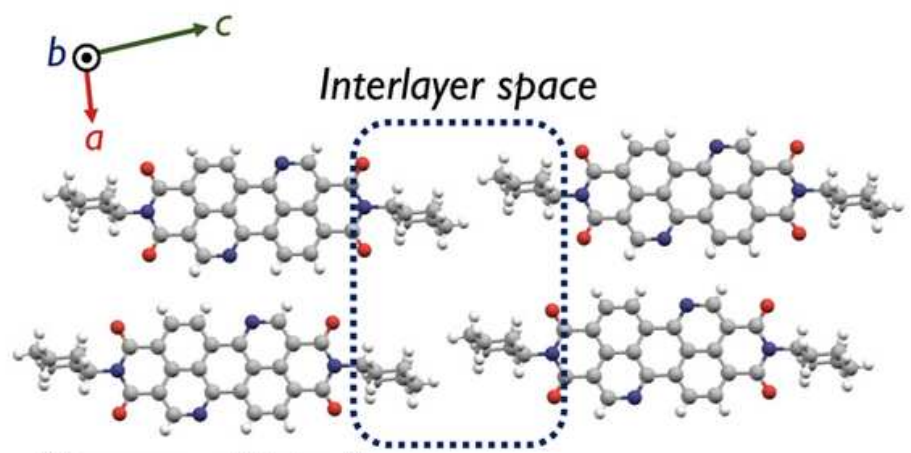

Absence of interlayer interactions
Enhanced LUMO overlaps

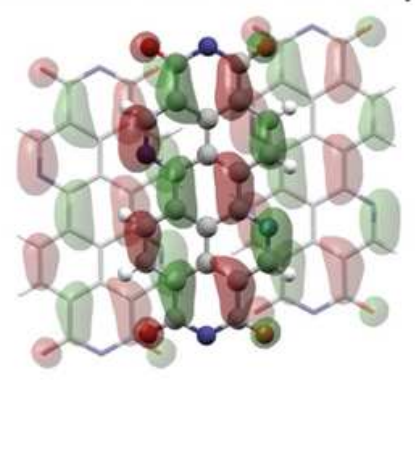

Multiple phenyl-to-
phenyl interactions

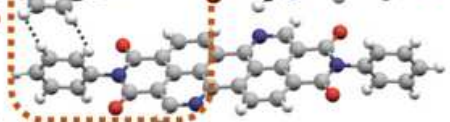

Multiple phenyl-to-
phenyl interactions

C

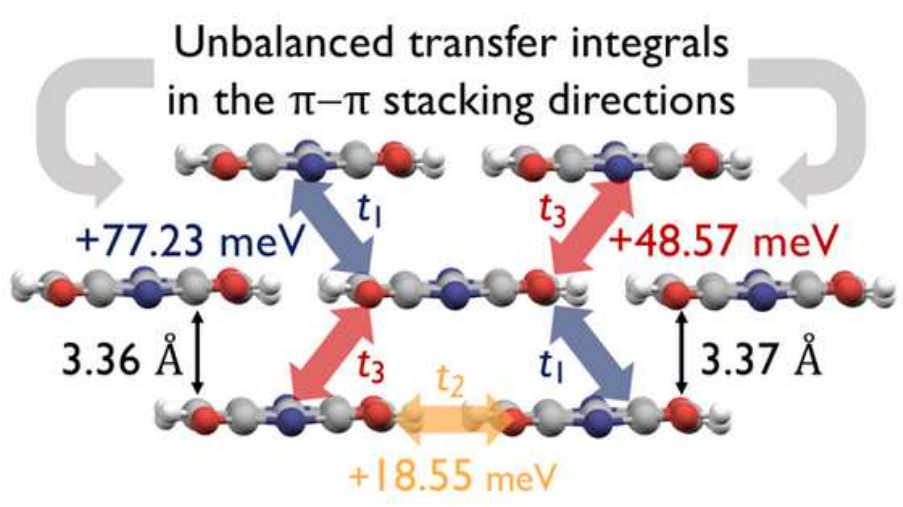

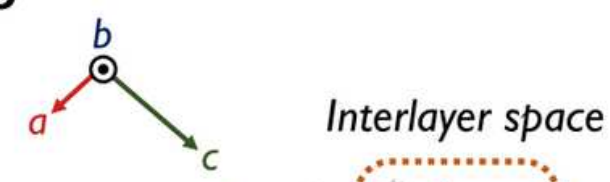
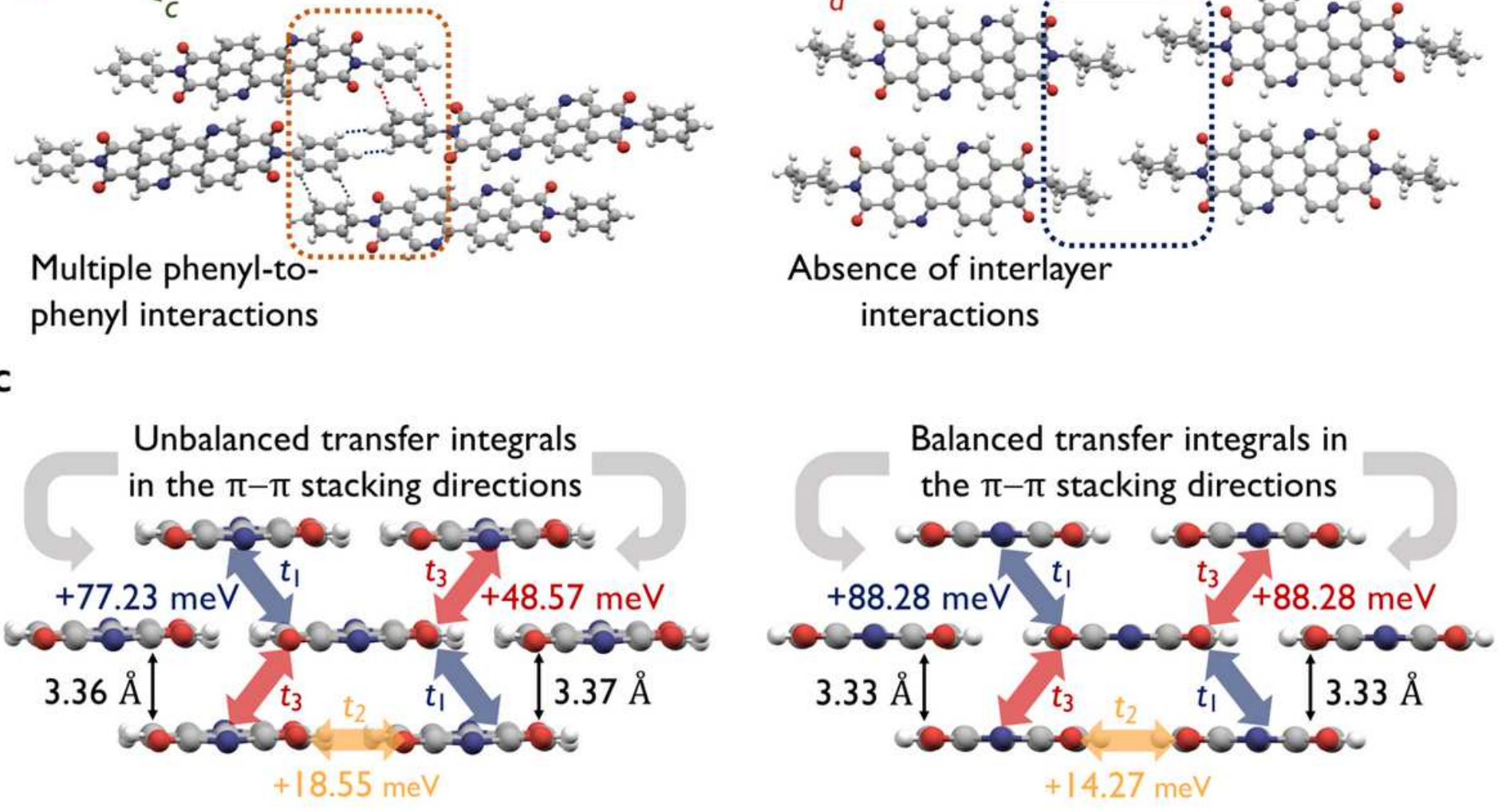

Figure 3

Molecular assemblies and charge-transport capabilities of Ph-BQQDI and Cy6-BQQDI. a Dimer distances, interactions and LUMO overlaps; b Interlayer interactions; c Stacking distances and transfer integrals. 

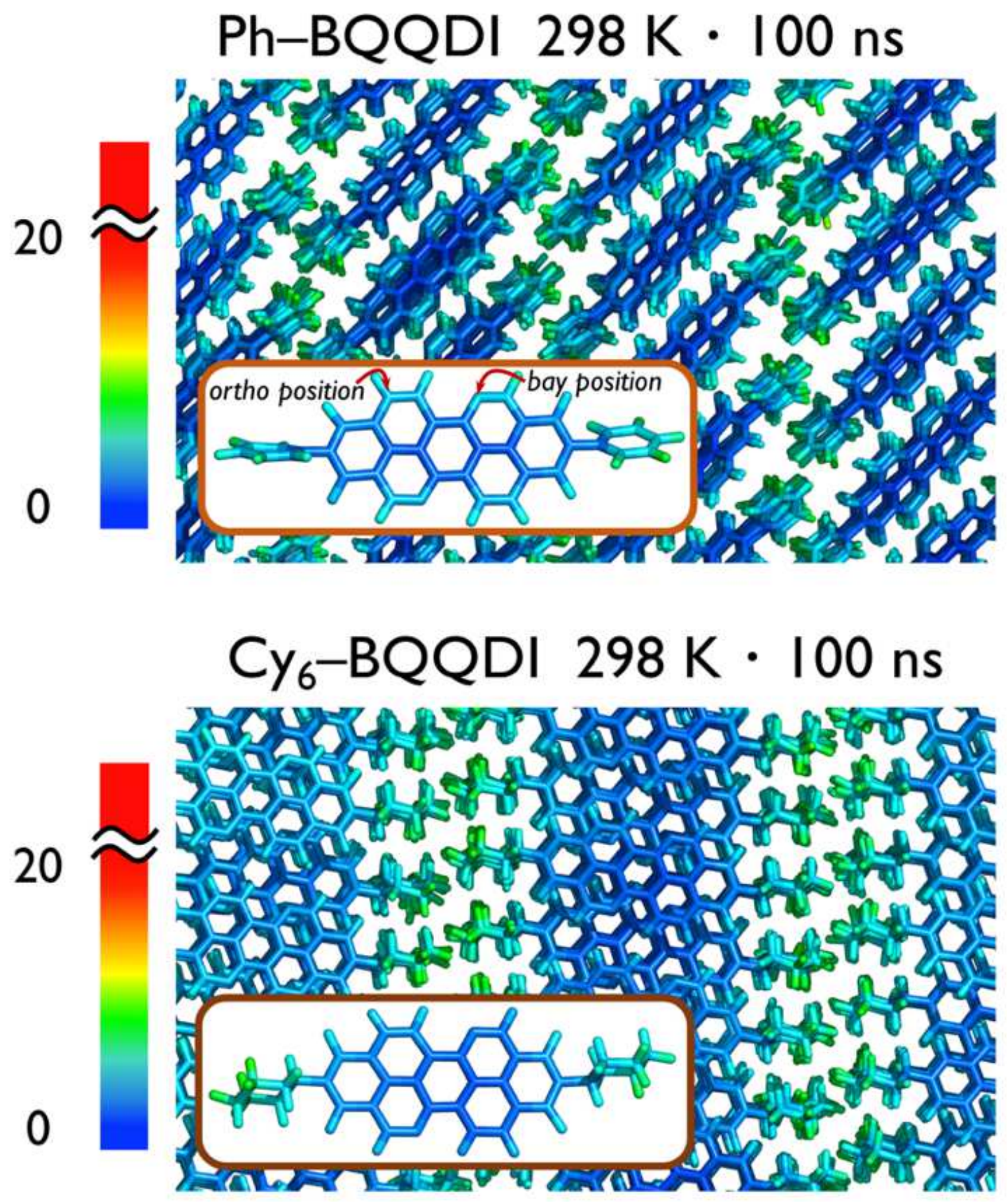

Figure 4

Molecular simulations of Ph-BQQDI and Cy6-BQQDI. Color-coded B-factor distributions obtained from the trajectories during the last $10 \mathrm{~ns}$ of a $100 \mathrm{~ns}$ MD simulations in the NTP ensemble. 


\section{a}

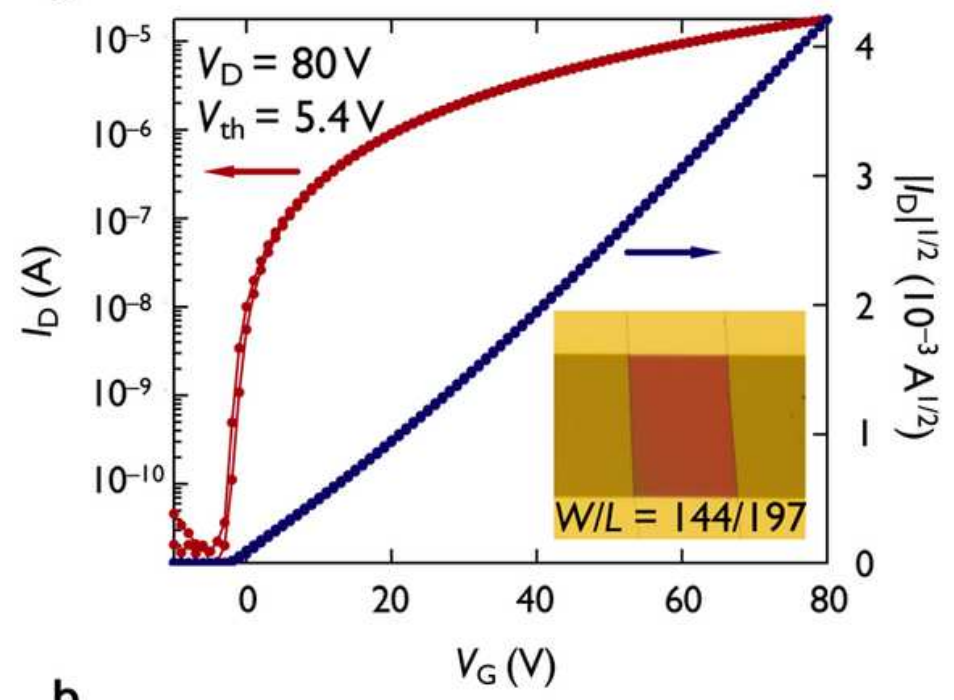

b
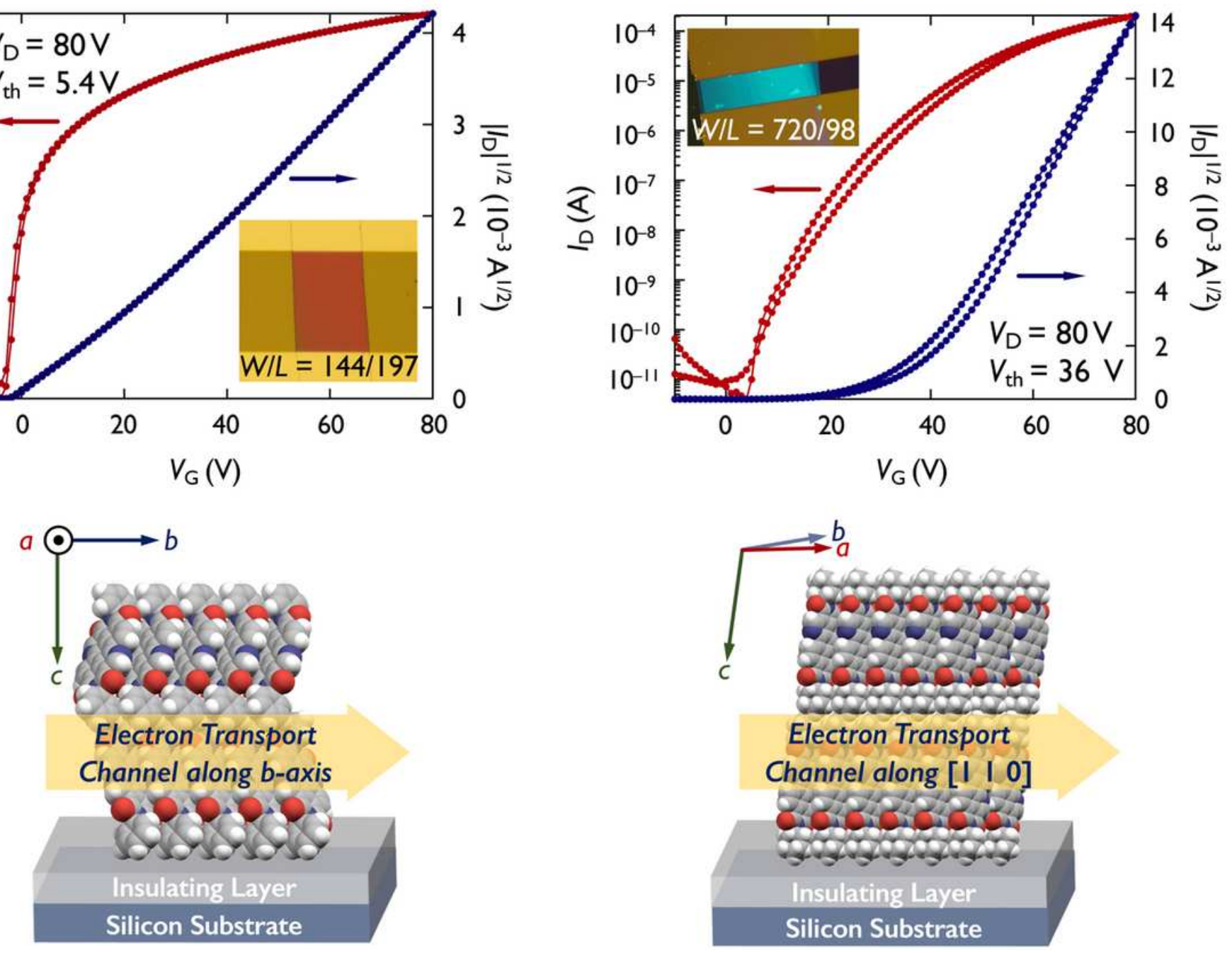

Figure 5

OFET performances and thin film assemblies of Ph-BQQDI (left) and Cy6-BQQDI (right). a Transfer characteristic of single-crystalline transistors; $b$ Molecular assemblies in device states and corresponding channel directions. 


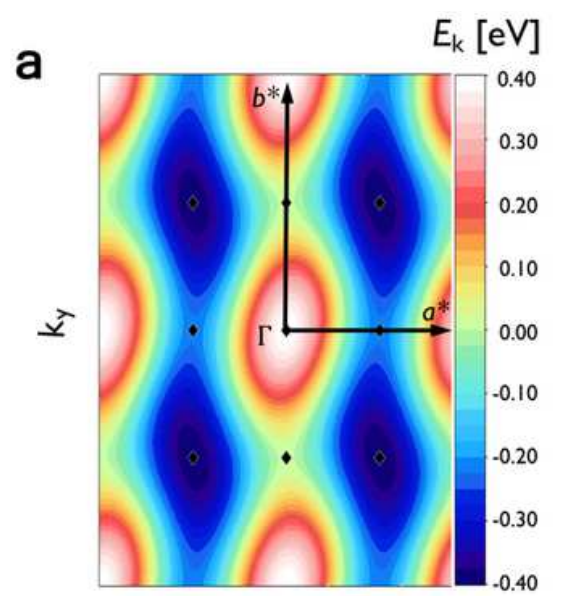

$k_{x}$

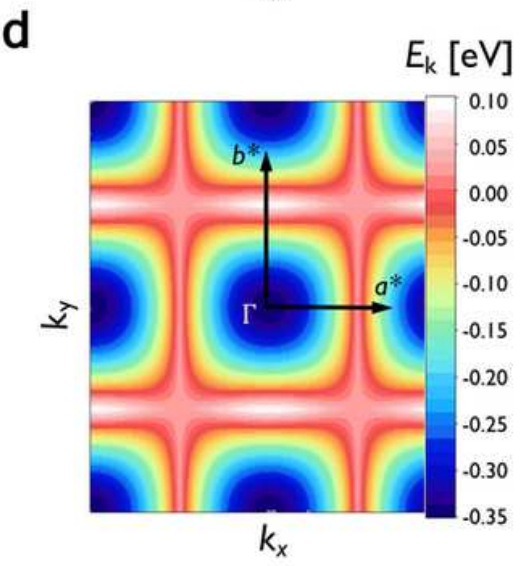

b

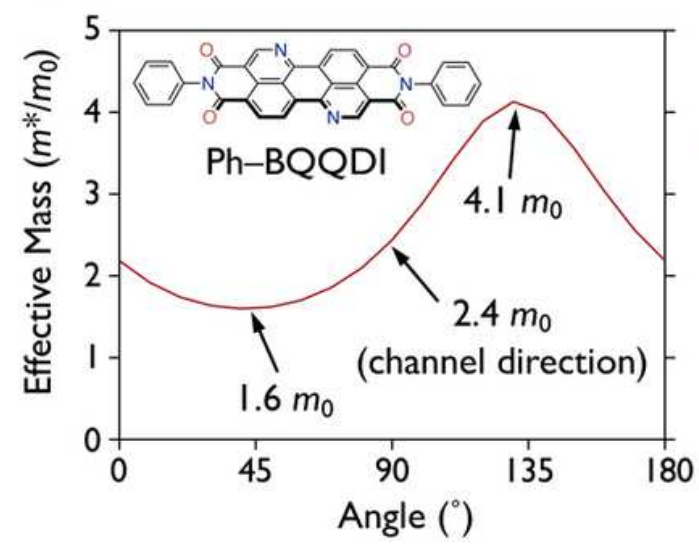

e

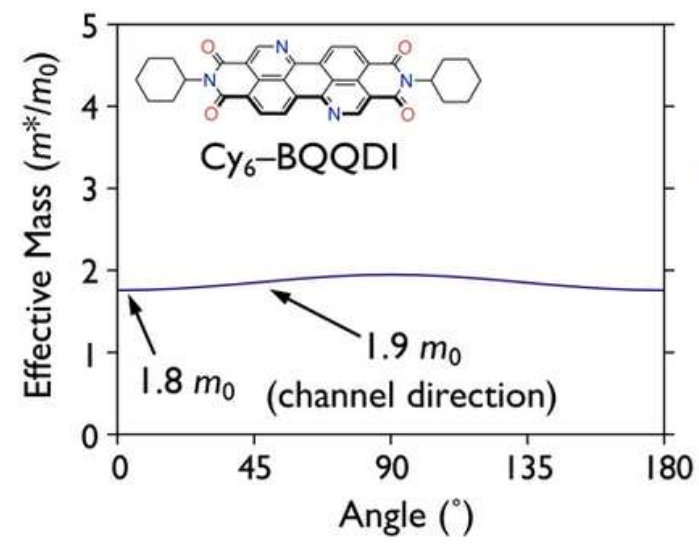

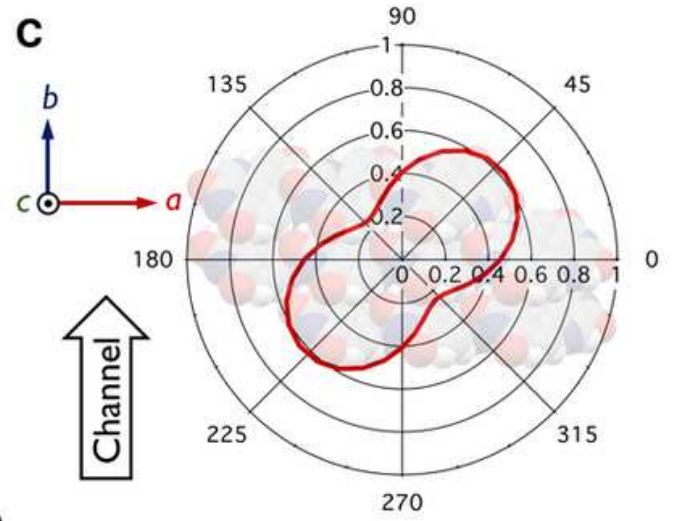

- $1 / m^{*}$ of Ph-BQQDI

f

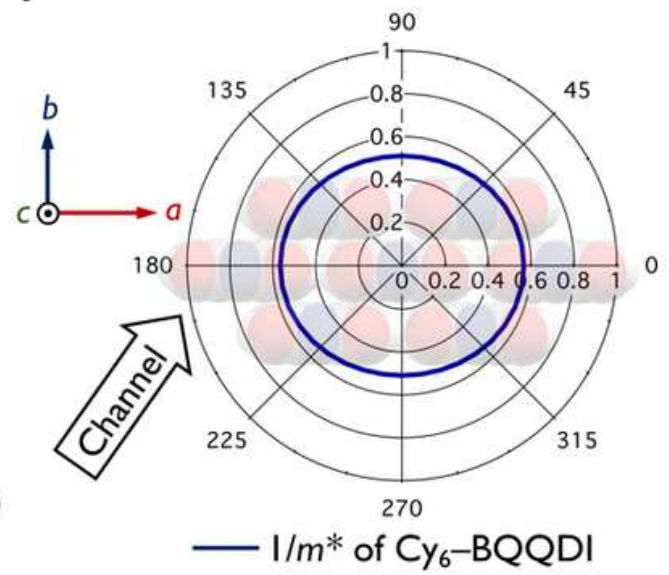

Figure 6

Charge-transport capabilities calculated by the tight-binding approximation. $a$ and d 2D HOMO band; $b$ and e Angle-resolved effective mass; $c$ and $f$ Angle-resolved inversed effective mass of Ph-BQQDI and Cy6-BQQDI, respectively.

\section{Supplementary Files}

This is a list of supplementary files associated with this preprint. Click to download.

- Cy6BQQDISICommunChem201015v5.pdf

- PhBQQDI.cif

- Cy6BQQDI.cif 